This influence persists in early neonatal period and might predispose SGA infants to metabolic syndrome in adulthood.

380

\section{DIETARY ARG-GLN DIPEPTIDE OR DHA REDUCES PATHOLOGICAL NEOVASCULARIZATION (NV) AND PROMOTES RETINAL VESSEL RE-GROWTH (VR) IN MOUSE MODEL OF ROP}

\section{S. Li Calzi ${ }^{1}$, L. Shaw ${ }^{1}$, N. Li ${ }^{2}$, J. Busik ${ }^{3}$, K. Morris ${ }^{4}$, J. $\mathrm{Neu}^{2}$, M.B. Grant ${ }^{1}$ \\ ${ }^{1}$ Department of Pharmacology and Therapeutics, ${ }^{2}$ Department of Pediatrics, University of Florida, Gainesville, FL, ${ }^{3}$ Department of Physiology, Michigan State University, East Lansing, MI, ${ }^{4}$ Global Discovery, Mead Johnson Nutrition Company, Evansville, IN, USA}

Aims: ROP is the leading cause of blindness in children. The most abundant sphingolipid in retinal membranes, sphingomyelin (S), is converted to ceramide $(\mathrm{C})$ by acid sphingomyelinase; $\mathrm{C} / \mathrm{S}$ ratio indicates inflammation and retinal injury. We compared the effects of Arg-GIn dipeptide, alone or with $\mathrm{DHA}$ on NV, VR and C/S ratio in a model of ROP.

Methods: 7-day old pups and their nursing dams were placed in $75 \%$ oxygen for five days. Pups were returned to room air on postnatal day $12\left(\mathrm{P}_{12}\right)$ and supplemented by oral gavage twice daily with ArgGIn (5 g/kg/d), DHA (2.5 g/kg/d), Arg-GIn + DHA, or vehicle through $\mathrm{P}_{17}$. On $\mathrm{P}_{17}$, pups were sacrificed and perfused with FITC-labeled dextran. Retinas were assessed for $\mathrm{NV}$ by enumeration of pre-retinal endothelial cells, intra-retinal vascular density (VR) or $\mathrm{C} / \mathrm{S}$ ratio by $\mathrm{MS}$.

Results: Compared to vehicle, pre-retinal NV was reduced by $61 \%$ and $51 \%$ with Arg-GIn or DHA ( $p<$ 0.05 both), with a $69 \%$ reduction observed for ArgGIn + DHA $(p<0.05)$. All treatments reduced vasoobliteration vs. vehicle ( $32 \%$ vs. $5.2 \%, p<0.05$ ). Arg-GIn, alone or with DHA, promoted intra-retinal vascular density (VR) vs. vehicle $(73-76 \%$ vs. $53 \%)$. Arg-GIn returned retinal C/S ratio to that of control.

Conclusion: These results demonstrate the dietary supplementation with Arg-GIn, alone or with DHA, reduces pre-retinal NV and promotes VR in a model of ROP, underscoring the importance of nutrition in modulating disease course. In addition, C/S ratio was normalized by Arg-GIn, supporting a key role for Arg-GIn in reducing acid sphingomyelinase.

381

LANGUAGE AND BEHAVIOUR FOLLOWUP FROM THE DINO (DHA FOR THE IMPROVEMENT OF NEURODEVELOPMENTAL OUTCOME IN PRETERM INFANTS) TRIAL

C.T. Collins ${ }^{1,2}$, L.G. Smithers ${ }^{1}$, L.A. Simmonds ${ }^{1}$, R.A. Gibson ${ }^{3}$, A.J. McPhee ${ }^{4}$, M. Makrides ${ }^{1,2}$

${ }^{1}$ Child Nutrition Research Centre, Women's and Children's Health Research Institute, North Adelaide, ${ }^{2}$ Discipline of Paediatrics, ${ }^{3}$ School of Agriculture, Food and Wine, The University of Adelaide, ${ }^{4}$ Neonatal Medicine, Children, Youth and Women's Health Service, Adelaide, SA, Australia

Background: Higher-dose docosahexaenoic acid, DHA, $(\sim 1 \%$ total fats) compared with standarddose $(\sim 0.3 \%)$ in infants born $<33$ weeks gestation improved the mental development of girls (JAMA, 2009). We aimed to evaluate the effect of higherDHA milk on behaviour and language development in early childhood.

Method: Follow-up of a subgroup of infants $(n=143)$ enrolled in the DINO trial. DINO was a multicentre randomised controlled trial, $\mathrm{n}=657$, with stratification for sex, birth weight $(<1250 \mathrm{~g}, \geq 1250 \mathrm{~g})$ and centre. Lactating women took tuna oil capsules (higherdose DHA) or soy oil capsules (standard); preterm infant formula with matching DHA composition was given if needed.

Language was assessed using the MacArthur Communicative Development Inventory (MCDI) at 26 months corrected age (CA). The Strengths and Difficulties Questionnaire (SDQ) and Short Temperament Scale for Children (STSC) were used to assess behaviour at 3-5 years CA. Results: MCDI scores did not differ between groups (Vocabulary Production: higher-DHA (mean $\pm S D$ ) 308 \pm 79 ; control 316 $\pm 92 ; P=0.8)$. Overall, there was a higher incidence of behavioural difficulties in these children compared with term-born children. However, the SDQ and STSC composite scores did not differ between groups (SDQ total difficulties: higher-DHA 10.3 \pm 6.0 ; control 9.5 \pm 5.5 ; $P=0.5$; STSC score: higher-DHA 3.1 \pm 0.7 ; control 3.0 $\pm 0.7 ; P=0.3$ ).

Conclusions: In this subgroup of infants, feeding preterm infants milk containing 3 times the standard DHA level did not result in any clinically meaningful change to language development or behaviour in early childhood. 